\title{
Unsupervised Pattern Recognition of Physical Fitness Related Performance Parameters among Terengganu Youth Female Field Hockey Players
}

\author{
M. R. Razali ${ }^{\#}$ N. Alias ${ }^{*}$, A. B. H. M. Maliki", R. M. Musa\#, L. A. Kosni ${ }^{\#}$, H. Juahir ${ }^{\%}$ \\ ${ }^{\#}$ Faculty of Applied Social Science, Universiti Sultan Zainal Abidin, 21300 Gong Badak, Terengganu, Malaysia \\ E-mail:mdrazali896@gmail.com,bisyrihusin84@gmail.com,rabiumuazu86@gmail.com,azuralaila@yahoo.com \\ *Faculty of Health Sciences, School of Rehabilitation Science, Universiti Sultan Zainal Abidin, 21300 Gong Badak, Terengganu, Malaysia \\ E-mail: norzulaikaalias@unisza.edu.my \\ ${ }^{\%}$ East Coast Environmental Research Institute (ESERI), Universiti Sultan Zainal Abidin, 21300 Gong Badak, Terengganu, Malaysia \\ E-mail: hafizanj@gmail.com
}

\begin{abstract}
This study aims to identify the most significant physical fitness parameters among youth female Terengganu field hockey players. Multivariate methods of unsupervised pattern recognition of principal component analysis (PCA) and descriptive statistic were used to determine the most significant physical fitness related performance parameters on 42 Terengganu youth female field hockey players. The first PC's project high factor loading in BMI (0.86) and predict $\mathrm{VO}_{2} \mathrm{max}(-\mathbf{0 . 8 2})$ as the most significant parameters indicating the requirements of body composition in this sport. The second PC's displayed high factor loading in 1-minute sit up (0.89) and 20-meter speed (-0.84) highlighting the need for core muscle strength. The third PC's demonstrated high factor loading in $\mathrm{V}$-sit and reach $(0.71)$ and maximum push up $(0.82)$ were recognising the importance of upper muscle strength in the sport. The results from the current study revealed that certain physical fitness components are seemed to be more pronounced in the performance of the game by the Terengganu female youth hockey players. The study has indicated that body composition, core muscle strength, and upper muscle strength are the most outstanding physical fitness variables possess by the players for the enactment of the game compared to other fitness parameters. Highlighting the physical fitness performance related parameters might help to evaluate the strength and weakness of the players on the relevant parameters which could prompt to the adjustment of the training programme for the inclusive improvement of the players.
\end{abstract}

Keywords - physical fitness parameters; body composition; field hockey; unsupervised pattern recognition

\section{INTRODUCTION}

Field hockey is a competitive, high-intensity, intermittent game that involves walking, jogging, sprinting in varied directions with and without ball [1]-[3]. In the field hockey, acceleration and deceleration occur concurrently resulted from the rapid changes in speed during the game. Thus, it demands good aerobic and anaerobic capacity to provide quick recovery process following high-intensity efforts among players and maintain work rates towards the end of the game [4], [5].

Moreover, the features of the game themselves required the involvement of both upper limbs and lower limbs to work simultaneously in different actions. For example, the upper limbs are required to hit hockey ball or hold the hockey stick while the lower limbs contracted continuously to maintain in semi-crouched posture during the game. Thus, the engagement of the whole body in this sport imposed high demands on the aerobic and anaerobic system [6]. Altogether, excellent physical fitness level could have a great impact on field hockey performance.

In addition, the previous study described that the speed, muscular strength, power, and agility are also prerequisite to produce high-performance game [7]. There is a study recognised the requirement of physical fitness parameters such as aerobic, anaerobic, muscular strength and power as significant and crucial in the game of field hockey [8]. In addition, it has been reported that the game of field hockey has similarity with the other sports such as ice hockey, basketball, lacrosse, and rugby in terms of physical fitness requirements and its characteristics. It displayed a high number of repeated near-maximal efforts during the game [9]. This finding has been confirmed by a recent study. It 
suggested that the nature of this game requires a high demand of physical fitness especially aerobic and anaerobic capacity [10]. This is because players are needed to maintain fit and powerful in $70 \mathrm{~min}$ of high-intensity competition [11]. Furthermore, physical fitness plays an important role in many aspects of sport especially in field hockey because of its capability to enhance the performance, reduce fatigue, prevent injury and ensure the cognitive system to function optimally [12]. The performance is increasing as the speed of the players increased. Several studies have reported that sprinting ability is attributed to high performance in field hockey. In this study, it has been reported that performance of field hockey with speed ability has been supported by a time-motion analysis study. It found that the elite players spent $22 \%$ of the total game employing short duration $(5.2 \pm$ $0.6 \mathrm{~s}$ ) with the high-intensity game. Therefore, it reflects that speed ability is one of the physical fitness parameters that are able to enhance game performance [13]. Numerous studies have been done on soccer and field hockey integrated multilaterally (e.g. anthropometric, physiological and psychological) variables of the players with the performance which revealed the important functional (physiological) characteristics of players in terms of aerobic endurance, anaerobic (running speed) and a combination of anaerobic and aerobic endurance which labelled as speed-endurance. It might be concluded that physiological characteristics of the players attribute to the high achievement in the sports performance [14], [15]. However, the most significant physical parameters related performance of female field hockey players in Terengganu is still not observed. Consequently, coaches will not be able to estimate the performance level and monitor the progression of the players.

Physical characteristics of players include size, physique, and body composition influence the performance in many sports [16], [17]. Thus, numerous studies have been conducted to analyse body composition of players on their performance [18]. Based on these studies, it revealed a clearer understanding on how nutritional and training programme can affect the physical performance of the players. However, there is less information exists for female field hockey players in Malaysia, especially in Terengganu describing physical fitness related performance characteristics. Thus, examination of physical fitness status in the field hockey players can contribute to specified training programs for the enhancement of the performance. In addition, several studies have assessed some of the physical fitness parameters of female field hockey players, but less study has observed the most important parameter that contributed to optimal performance on the same population in Terengganu. Therefore, this study aims to identify the most significant physical fitness parameters among youth female Terengganu field hockey players.

\section{MATERIAL AND METHOD}

\section{A. Participants}

42 Terengganu youth female field hockey players (age, $17.5 \pm 1.59$ years; weight, $53.12 \mathrm{~kg} \pm 6.41 \mathrm{~kg}$; height, 156.01 $\mathrm{cm} \pm 5.05 \mathrm{~cm}$ ) from SUKMA participated in the study. Participants criteria were as follows: (age, $17.5 \pm 1.59$ years), weight $(53.12 \mathrm{~kg} \pm 6.41 \mathrm{~kg})$, height $(156.01 \mathrm{~cm} \pm 5.05 \mathrm{~cm})$.
They were informed about the procedure of the study before the signed the informed consent. The ethical committee of the University Sultan Zainal Abidin approved the procedure. All the participants were tested in five different tests: speed test, multistage fitness test shuttle run, V sit and reach test, maximum push-up test, and 1-minute sit-up test. Anthropometric measurements were taken for height and weight. All the data were collected at Terengganu Sports Council hall.

\section{B. Design}

In order to identify the most significant physical fitness parameters among youth female Terengganu field hockey players, an ex-post facto design was employed. The process of data collection was conducted during the off day training of the players. All the measurements were completed on the same day, so the training schedule of the athletes was no affected. In addition, the commitment of the players was not required as this study was not implemented any intervention or rehabilitation protocols. Thus, this study has no control over the players. The advantage of this study is it allows the researchers to assess current physical fitness level of the players.

\section{Anthropometric Measurements}

Height and weight were measured by using a standard protocol. Standing height was measured with shoes off, feet together and the highest point of the head by using a standard wall-mounted stadiometer. The readings were taken to the nearest $0.5 \mathrm{~cm}$. Weight was measured to the nearest $0.5 \mathrm{~kg}$ using a calibrated scale (Seca, Hamburg, Germany).

\section{Multistage Fitness Test Shuttle Run test}

The Multistage Fitness Test Shuttle Run is a valid and reliable test to determine $\mathrm{VO}_{2}$ max in team sports athletes [19], [20]. It consists a series of 20-m shuttle runs in which the speed increases progressively. Participants are needed to maintain the given speed until reaching volitional fatigue. The test involves continuous running between two lines 20 $\mathrm{m}$ apart in time to recorded beeps. They reached the line before the beep sounds. The test will be terminated if the participants fail to reach the line for two consecutive ends [21]. Participants were instructed to perform warming up and stretching exercises before the test. Then, they were asked to do cooling down following the completion of the test [21]. The number of shuttles completed by the participants was recorded, and an equivalent $\mathrm{VO}_{2}$ max was calculated [22]. The validity of this test has previously been reported to provide high accuracy in estimation of VO2 $\max$ [21].

\section{E. Speed Test}

Speed was assessed by using 10 and $40 \mathrm{~m}$ speed tests. A stopwatch was used to record the time of running. Subjects were stand in stationary position at the starting line. Three $10-\mathrm{m}$ and $40-\mathrm{m}$ trials were performed by each participant. The times recorded were calculated for the average.

\section{F. Maximum Push Up Test}

The maximum push up is purposely to measure the muscle endurance. This test was followed the standard protocols. The participants were laid down essentially. The participants' hands were in line with the shoulders and backs 
were kept straight throughout the test. They lowered their body until the chest touched the clenched fist of the counter, who held their fists in line with the sternum. Then, the arms were straightened immediately. Only the hands and feet were in contact with the floor. The participants were not allowed to put their knees on the floor if they stopped during the test. The test was considered as failed if they did not comply with it. No score was given if the performance was not met the standards. The score was given for the participants who completed the test without any failure and followed standard procedures.

\section{G. 1 Minute Sit Up Test}

Core strength is significant in controlling the body movement optimally. Sit-ups test is performed to assess the core strength based on its reliability and validity. The participants lay on their backs with their knees were at $90^{\circ}$ with the hands straight out in front resting on the thighs as the starting position. The test was started by the participants crouched their body into a $30^{\circ}$ such that the hands slid to on top of the knees and then returned to the starting position. During the procedure, a partner held the players' feet put on the ground for stabilization [23]. Players

sat up until they touched their knees to both elbows, then they came back to the floor. Sit up was considered as complete and given one repetition if the shoulder blades touched the ground. No score was given if the performance was not met the standard procedures and there were no half counts. The participants completed the test within 1 minute.

\section{H. V Sit and Reach Test}

Field hockey demands good flexibility. The assessment of the erector spinae and hamstring flexibility becomes an important part of fitness and sports assessment for many times. Flexibility has good potential to reduce the risk of injury during the game. In this study, a standard flexibility protocol, $\mathrm{V}$ sit and reach test was used. The participants started the test by sitting on the floor with extended knees. The arms were extended forward with palms facing down. The participants reached forward with both hands and sustained this position for 10 seconds. The knees must be in contact with the ground and were not allowed to be raised. The position of maximum reach will be recorded. The test was repeated three times for each participant, and the average was calculated for the final result. A warm up routine was performed before the test was conducted.

\section{Data Analysis}

Finally, all data matrixes of $(42 \times 9=378)$ were measured and presented as mean \pm SD values (see Table 1 ). Before main statistical analysis, the total of missing data, data error, and outlier were checked in order to expedite the data analysis [24]. The normality of data distribution, as well as the outliers, were also checked using Kolmogorov-Smirnov and box-plot method [25].

$$
Z_{i j}=\left(X_{i j}-\mu\right) / a
$$

whereas $Z i j$ is the $j t h$ value of the standardize score of the measured variable $i$, meanwhile $X i j$ is the observation of $j t h$ on the variable $i ; \mu$ and $\sigma$ are the mean and the standard deviation of $X i j$, respectively.

In this current study, Principal component analysis (PCA) was employed as the main statistical analysis to find out the most significant variables related to field hockey. By using this analysis as expressed in the equation (1), it helps to reduce the less significant data set which is considered as one of the most rampant and advantageous statistical methods for recognition the latent structure of a set of variables with a minimal loss of original data [26].

\section{RESULTS AND DISCUSSION}

Prior data analysis, Kaiser-Meyer-Olkin measure of sampling adequacy (0.535) and Bartlett's test of sphericity ( $p$ $=0.001)$ reported that data meet the assumptions of the sampling adequacy and the significant correlation between variables for further analysis. Table 1 exhibits the descriptive statistics of participants characteristics as projected as mean and standard deviation (SD) values for all variables.

The objective of the present study is to identify the most significant physical fitness parameters among youth female Terengganu field hockey players. A total number of 42 Terengganu youth female field hockey players were purposely recruited for this study. Standard battery fitness and body composition tests were conducted, and an unsupervised pattern recognition of principal component analysis (PCA) and descriptive statistic were employed to determine the most significant physical fitness related performance parameters in field hockey.

TABLE I

DESCRIPTIVE STATISTICS OF PARTICIPANTS

\begin{tabular}{|l|l|l|c|}
\hline Statistic & M & SD & Participants \\
\hline Age & 17.50 & 1.59 & 42 \\
\hline Weight (KG) & 53.12 & 6.41 & 42 \\
\hline Height (M) & 156.01 & 5.05 & 42 \\
\hline BMI & 21.81 & 2.35 & 42 \\
\hline V sit and reach (cm) & 48.96 & 11.72 & 42 \\
\hline Sprints 20 (s) & 3.60 & 0.47 & 42 \\
\hline Max push up & 39.64 & 13.50 & 42 \\
\hline 1 Minute sit up & 34.52 & 9.92 & 42 \\
\hline Predicted vo2max & 37.92 & 9.11 & 42 \\
\hline
\end{tabular}

In the current study, VFs with absolute values greater than 0.70 were standardized as the selection threshold. It describes moderate to strong loadings on the extracted factors. Based on Table 2, the first PC's demonstrated high factor loading in BMI (0.86) and predicted VO2max (-0.82). Thus, the present study suggested that body composition is the most significant parameter in the field hockey. This finding agrees with another study that found the significance effects of variation in body composition toward functional capacities and performances of athletes in many sports [27]. This result is in accordance with the studies mentioned that increased body fatness was associated with poorer performance in aerobic and anaerobic capacities in the players [28], [29]. In addition, the previous study that has examined the effect of body composition and performance in cross-country skiers has found that a higher muscle mass percentage is associated with maximal consumption $\left(\mathrm{VO}_{2}\right.$ 
max) and late lactate threshold, especially the beginning of blood lactate accumulation (OBLA) [30], [31]. As a lean body mass increased, muscle mass also increased and therefore positively influences endurance. However, a gain in body fat negatively influences endurance [32]. These studies suggested that body composition should be considered by the nature of team sports. As we know that, certain sports such as field hockey demanding high levels of relative strength, agility, speed, and endurance. Thus, body composition can be a potential factor affecting these functional components in positive ways [28], [29]. Furthermore, body composition such as body mass is an important factor for successful performance in the field hockey. This is because a study suggested that increased body mass inappropriately resulted in the reduction of running fitness. This finding has been confirmed by a study that has reported a strong relationship between body mass and running velocity. It suggested that high body mass caused lower running velocity at lactate threshold and exhibited a reduction of running ability over $10-\mathrm{km}$ distances [33]. Body composition may reduce the running speed of female field hockey players. Thus, this current study suggests field hockey does not necessarily require high body weight for maximal performance. The results of this study revealed that ideal BMI contributed to high performance during the game. In addition, the results have satisfied the findings from previous studies which found that body composition may influence the player's level of physical fitness related performance in the female athletes. In the field hockey, the players have to cover a long distance of area. The players need to carry their body weight during the game by the facilitation of a large composition of active tissue (muscle) in relation to a small composition of fat tissue. However, a high body weight of the players will result in difficulty and reduce capability for them to maintain their physical fitness level until the end of the game. Therefore, this present study recommends it is necessary to employee good ideal BMI in order to achieve high performance in the field hockey [34].

TABLE II

FACTOR LOADINGS AFTER VARIMAX ROTATION

\begin{tabular}{|l|l|l|l|}
\hline & VF1 & VF2 & VF3 \\
\hline BMI & 0.868 & 0.005 & 0.137 \\
\hline V sit and reach (cm) & 0.470 & 0.027 & 0.714 \\
\hline Sprints 20 (s) & 0.070 & -0.842 & 0.250 \\
\hline Max push up & -0.239 & -0.097 & 0.825 \\
\hline 1 Minute sit up & -0.093 & 0.885 & 0.139 \\
\hline Predicted vo2max & -0.819 & 0.219 & 0.189 \\
\hline Eigenvalue & 1.961 & 1.348 & 1.284 \\
\hline Variability (\%) & 28.596 & 25.847 & 22.099 \\
\hline Cumulative \% & 28.596 & 54.442 & 76.541 \\
\hline
\end{tabular}

The second PC's displayed high factor loading in 1minute sit up (0.89) and 20-meter speed (-0.84). The results of this study demonstrated the importance of core muscle strength in the field hockey. According to [35], core strength is where the muscles or muscle group produce force maximally at a specific velocity. Similarly, the finding is in concordance with the definition of core strength derived from another study. They described it as the ability of muscles to generate force through contractile forces and intra-abdominal pressure [36]. Core muscle strength can produce high force and lead the muscles of the shoulders, arms, and legs to contract effectively [35].

As a result, it can again reduce the risk of injury and increase sports performance in terms of speed, agility, power and aerobic endurance. Therefore, it will lead the body functioning to optimal level [37] and enhance great athletic performance especially in the field hockey [38]. Moreover, studies have shown that core strength can provide better stability and muscles controlling such as abdominal, paraspinal and gluteal during the games. Therefore, it can prevent any injury especially in spinal or extremities among the athletes. The lower back injury is the second and third most common injury in practice, in women's gymnastics, field hockey, and volleyball as demonstrated by a previous study [39]. Another study reported 8\% more female athletes had lower back pain than male athletes [40]. Therefore, this study suggests that core muscle strength is important to prevent lower back injury. In addition, it significantly influences the postural maintenance and distribution and absorption of force in the body [41]. The contraction of core muscles controls the actions of the trunk and lower limbs. They are heavily loaded during the game as they maintained the postural stability of the players. The unnatural semicrouched posture and unequal distribution of body weight cause an impact on body posture of the players [42]. These significant findings confirmed what had been emphasized in previous research on field hockey players. In this sports activity, the players are to flex the trunk forward to the ground for the maximum groundwork and to cover a wider range [43]. Finally, the maximum strain of muscles specifically abdominal muscles, as well as back muscles, will be developed during the entire length of the game [44]. Therefore, the important findings of the current study present that the core muscle strength is highly demanded in the field hockey. In addition, the present findings highlight the important of this muscle in preventing the muscle strain.

The third PC's demonstrated high factor loading in V-sit and reach (0.71) and maximum push up (0.82). The results of this analysis clearly showed the need for upper muscle strength in the field hockey. A study demonstrated that upper body strength and power might contribute to the success in many sports with great performance. It also mentioned that players with superior upper body anaerobic power might achieve success in the games [45], [46]. Upper muscle strength and power are particularly important in a certain sport such as rugby league, soccer, and field hockey which involve frequent contact, collision, and attacks from opponents. In the field hockey, it demands the player ability to tackle an opponent, execute skills and to hold and hit the balls for goal scoring [47]. Analysis and assessment of upper muscular strength have to be done in handball players, and the results showed that muscular strength played an important role influencing throwing handball velocity. In addition, it demonstrated a strong relationship between throwing velocity and elbow extension isokinetic torque [48] and maximal isometric handgrip strength [49]. The derived findings from the study can be implemented in the field hockey as the nature of this game also requires good upper muscle strength. Therefore, in the field hockey, the muscular strength, as well as hand grip strength, is two related 
components that enable the players to carry the stick and hit the ball in high velocity for goals.

Based on the Fig. 1, it illustrated the correlations between all variables and factors loading after varimax rotation. It discovered the most important variables related to the demands of the field hockey game. Meanwhile, Table 3 projected the percentage of important variables across all variables measured. Thus, it can be concluded that the field hockey players require well-developed upper muscle strength.

To summarize the important of certain factors in enhancing the performance of the field hockey players, a current study has been adopted as references. The study has found that an elite professional soccer demands physical, physiological and psychological components and presents with a complex characteristic in the game [50].

The belief can be used to pronounce the significance of physical fitness parameters related performance in the field hockey because soccer and field hockey are two highintensity games that have similarities physiologically and require almost the same physical parameters. However, this present study only measured the physical fitness parameters in the performance of field hockey compared to other studies which measured the other components.

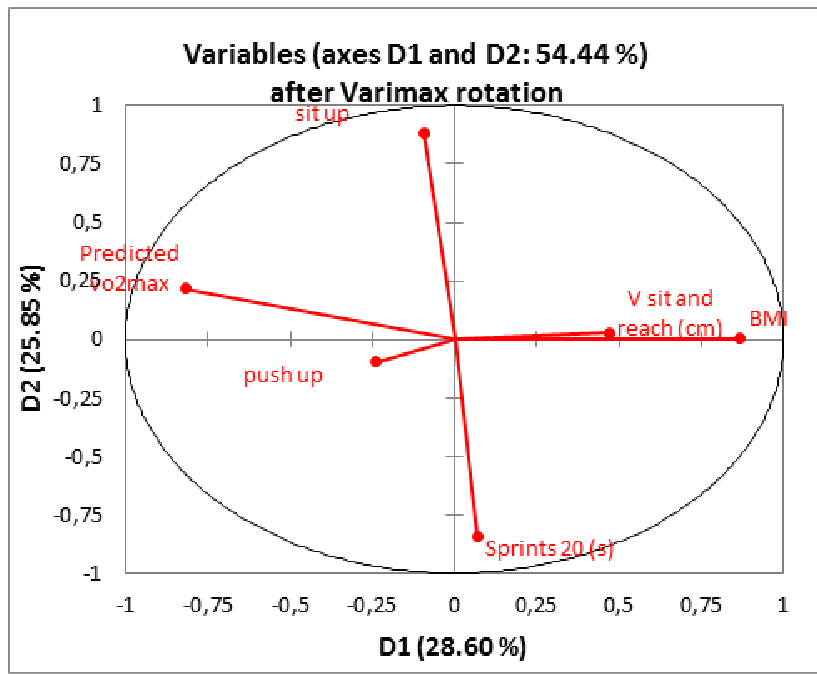

Fig. 1 Correlations between variables and factors after Varimax rotation

TABLE III

CONTRIBUTION OF THE VARIABLES (\%) AFTER VARIMAX ROTATION

\begin{tabular}{|l|l|l|l|}
\hline & VF1 & VF2 & VF3 \\
\hline BMI & 43.869 & 0.001 & 1.425 \\
\hline V sit and reach (cm) & 12.889 & 0.047 & 38.423 \\
\hline Sprints 20 (s) & 0.285 & 45.751 & 4.709 \\
\hline Max push up & 3.328 & 0.606 & 51.289 \\
\hline 1 Minute sit up & 0.501 & 50.515 & 1.457 \\
\hline Predicted vo2max & 39.128 & 3.080 & 2.697 \\
\hline
\end{tabular}

In the end, the analysis of this study showed that the most significant physical fitness parameters related performance are body composition, core muscle strength, and upper muscle strength. From these significance findings, it can be suggested that a training programme should focus on strengthening and endurance exercise which is believed to improve the physical fitness level in the female field hockey players in the future.

\section{CONCLUSION}

The current study has successfully revealed that certain physical fitness components are seemed to be more pronounced in the performance of the game by the Terengganu female youth hockey players. This finding of this study provides a greater understanding of the physical requirements of female field hockey players. This indicates that body composition, core muscle strength, and upper muscle strength are an essential physical fitness variables possess by the players for the enactment of the game compared to other fitness parameters. Highlighting the physical fitness performance related parameters might help to evaluate the strength and weakness of the players on the relevant parameters which could prompt to the adjustment of the training programme for the inclusive improvement of the players. Again, this study may suggest coaches of interventions or training programme that could be implemented to further improve the athlete's success and may assist them to estimate the athlete's potential to perform very well in the field hockey performance.

In conclusion, the present research highlighted the importance of recognizing the most significant physical fitness related performance variables in female field hockey athletes, since it represents a useful instrument for enhancing the sports performance in this sport. Moreover, this current study may be beneficial tools or methods for the coaches in the process of selecting the talented field hockey players in the future.

\section{ACKNOWLEDGMENT}

The authors gratefully thank the Terengganu Sports Council for its contribution and valuable suggestion on this research. The authors also sincerely and wholeheartedly thank all team managers, coaches, and players for their assist and commitment throughout the data collection process.

\section{REFERENCES}

[1] T. Hinrichs, J. Franke, S. Voss, W. Bloch, W. Schanzer, and P. Platen, "Total hemoglobin mass, iron status, and endurance capacity in elite field hockey players," Journal of Strength and Conditioning Research, vol. 1, pp. 629-638, Mar. 2010.

[2] J. Bloomfield, R. Polman, P. E. O'Donoghue, and L. A. McNaughton, "Effective speed and agility conditioning methodology for random intermittent dynamic type sports," Journal of Strength and Conditioning Research, vol. 1, pp. 1093-1100, Nov. 2007.

[3] M. T. Elferink-Gemser, C. Visscher, M.A. Van Duijn, and K.A. Lemmink, "Development of the interval endurance capacity in elite and sub-elite youth field hockey players," British Journal of Sports Medicine, vol. 40, pp. 340-345, Apr. 2006.

[4] H. MacLeod, C. Bussell, and C. Sunderland, "Time-motion analysis of elite women's field hockey, with particular reference to maximum intensity movement patterns," International Journal of Performance Analysis in Sport, vol. 1, pp. 1-12, May 2007.

[5] T. Reilly and D. Doran, "3 fitness assessments," Science and Soccer, vol. 8, pp. 21, Dec. 2003.

[6] T. A. Astorino, P. A. Tam, J. C. Rietschel, S. M. Johnson, and T. P. Freedman, "Changes in physical fitness parameters during a competitive field hockey season," Journal of Strength and Conditioning Research, vol. 18, pp. 850-854, Nov. 2004.

[7] T. Reilly and A. Borrie, "Physiology applied to field hockey," Sports Medicine, vol. 14, pp. 10-26, Jul. 1992.

[8] M. Spencer, S. Lawrence, C. Rechichi, D. Bishop, B. Dawson, and C. Goodman, "Time-motion analysis of elite field hockey, with special reference to repeated-sprint activity," Journal of Sports Sciences, vol. 22, pp. 843-850, Sep. 2004.

[9] J. Lythe and A. E. Kilding, "Physical demands and physiological responses during elite field hockey," International Journal of Sports 
Medicine, vol. 32, pp. 532-528, Jul. 2011.

[10] P. M. Boyle, C. A. Mahoney, and W. F. M. Wallace, "The competitive demands of elite male field hockey," Journal of Sports Medicine and Physical Fitness, vol. 34, pp. 235-241, Sep. 1994.

[11] J. F. Burr, R. K. Jamnik, J. Baker, M. Alison, G. Norman, and E. J. Mcguire, "Relationship of physical fitness test results and hockey playing potential in elite-level ice hockey players," Journal of Strength and Conditioning Research, vol. 22, pp. 1535-1543, Sep. 2008.

[12] F. Lothian and M. Farrally, "A time-motion analysis of women's hockey," Journal of Human Movement Studies, vol. 26, pp. 255-265, Sep. 1994.

[13] D. Bishop and M. Spencer, "Determinants of repeated-sprint ability in well-trained team sport athletes and endurance-trained athletes," Journal of Sports Medicine and Physical Fitness, vol. 44, pp. 1-7, Mar. 2004.

[14] R. Strzelczyk, J. Konarski, K. Karpowicz, and J. Janowski, "Changes in the main abilities of field hockey players during the preparatory period leading up to the main competition," Gymnica, vol. 31, pp. 1721, Dec. 2001.

[15] M. T. Elferink-Gemser, C. Visscher, K. Lemmink, and T. Mulder, "Relation between multidimensional performance characteristics and level of performance in talented youth field hockey players," Journal of Sports Sciences, vol. 1, pp. 1053-1063, Nov. 2004.

[16] T. Reilly, A. M. Williams, A. Nevill, and A. Franks, "A multidisciplinary approach to talent identification in soccer," Journal of Sports Sciences, vol. 1, pp. 695-702, Jan. 2000.

[17] C. M. Calo, S. Sanna, I. S. Piras, P. Pavan, and G. Vona, "Body composition of Italian female hockey players," Biology of Sport, vol. 26, pp. 23-31, Jan. 2009.

[18] J. Sproule, C. Kunalan, M. McNeill, and H. Wright, "Validity of the 20 MST for predicting $\mathrm{VO}_{2 \max }$ of adult Singaporean athletes," British Journal of Sports Medicine, vol. 27, pp. 202-204, Sep. 1993.

[19] L. A. Leger, D. Mercier, C. Gadoury, and J. Lambert, "The multistage 20 metre shuttle run test for aerobic fitness," Journal of Sports Sciences, vol. 6, pp. 93-101, Jun. 1988.

[20] L. A. Leger and J. Lambert, "A maximal multistage 20-m shuttle run test to predict VO2 max," European Journal of Applied Physiology and Occupational Physiology, vol. 49, pp. 1-2, Jun. 1982.

[21] J. Brewer, R. Ramsbottom, and C. Williams, Multistage Fitness Test: A Progressive Shuttle-Run Test for the Prediction of Maximum Oxygen Uptake, Canberra, Australia: Australian Coaching Council, 1988.

[22] H. Juahir, S. M. Zain, M. K. Yusoff, T. T. Hanidza, A. M. Armi, M. E. Toriman, and M. Mokhtar, "Spatial water quality assessment of Langat River Basin (Malaysia) using environmetric techniques," Environmental Monitoring and Assessment, vol. 173, pp. 625-641, Feb. 2011.

[23] M. R. Abdullah, R. M. Musa, A. B. H. M. Maliki, N. A. Kosni, and H. Juahir, "Intelligent Prediction of soccer technical skill on youth soccer player's relative performance using multivariate analysis and artificial neural network techniques," International Journal on Advanced Science, Engineering and Information Technology, vol. 6, pp. 668-674, Oct. 2016.

[24] M. R. Abdullah, A. B. H. M. Maliki, R. M. Musa, N. A. Kosni, and H. Juahir, "Multi-hierarchical pattern recognition of athlete's relative performance as a criterion for predicting potential athletes," Journal of Young Pharmacists, vol. 8, pp. 463-470, Aug. 2016.

[25] A. Andasuryani, Y. A. Purwanto, I. W. Budiastra, and K. Syamsu, "Determination of catechin content in gambir powder from dried gambir leaves quickly using FT NIR PLS model," International Journal on Advanced Science, Engineering and Information Technology, vol. 4, pp. 303-307, Aug. 2014.

[26] S. M. Collins, M. Silberlicht, C. Perzinski, S. Smith, and P. Davidson, "The relationship between body composition and preseason performance tests of collegiate male lacrosse players," Journal of Strength and Conditioning Research, vol. 28, pp. 2673-2679, Sep. 2014.

[27] F. Slinde, C. Suber, L. Suber, C. E. Edwén, and U. Svantesson, "Test-retest reliability of three different countermovement jumping tests," Journal of Strength and Conditioning Research, vol. 1, pp. 640-644, Mar. 2008.

[28] R. Maughan, "The athlete's diet: Nutritional goals and dietary strategies," Proceedings of the Nutrition Society, vol. 1, pp. 87-96, Feb. 2002.

[29] W. C. Fornetti, J. M. Pivarnik, J. M. Foley, and J. J. Fiechtner, "Reliability and validity of body composition measures in female athletes," Journal of Applied Physiology, vol. 1, pp. 1114-1122, Sep. 1999.

[30] M. Krzykała and P. Leszczyński, "Asymmetry in body composition in female hockey players," Journal of Comparative Human Biology, vol. 66, pp. 379-386, Aug. 2015.

[31] P. Larsson and K. Henriksson-Larsén, "Body composition and performance in cross-country skiing," International Journal of Sports Medicine, vol. 29, pp. 971-975, Dec. 2008.

[32] P. O. Astrand, K. Rodahl, and H. A. Dahl, Textbook of Work Physiology: Physiological Bases of Exercise, 4th ed., New York, USA: McGraw-Hill, 2003.

[33] R. J. Buresh, K. E. Berg, and J. M. Noble, "Relationship between measures of body size and composition and velocity of lactate threshold," Journal of Strength and Conditioning Research, vol. 3, pp. 504-507, Aug. 2004.

[34] M. K. Jain, "Body composition: Concept for coaches and physical trainers," Journal of Sports and Sports Sciences, vol. 27, pp. 48-57, Jan. 2004.

[35] G. J. Lehman, "Resistance training for performance and injury prevention in golf," Journal of the Canadian Chiropractic Association, vol. 50, pp. 27-42, Mar. 2006.

[36] M. D. Faries and M. Greenwood, "Core training: Stabilising the confusion," Strength and Conditioning Journal, vol. 29, pp. 10-25, Apr. 2007.

[37] M. J. Comerford, "Performance stability: Module 1 stability for performance-Course 1: Core stability concepts," Ludlow, England: Comerford and Performance Stability, 2007.

[38] G. D. Myer, K. R. Ford, and J. P. Palumbo, "Neuromuscular training improves performance and lower-extremity biomechanics in female athletes," Journal of Strength and Conditioning Research, vol. 19, pp. 51-60, Feb. 2005.

[39] R. Dick, J. Agel, and S. W. Marshall, "National collegiate athletic association injury surveillance system commentaries: Introduction and methods," Journal of Athletic Training, vol. 42, pp. 173, Apr. 2007.

[40] R. Ferber, L. Bolgla, J. E. Earl-Boehm, C. Emery, and K. HamstraWright, "Strengthening of the hip and core versus knee muscles for the treatment of patellofemoral pain: A multicenter randomized controlled trial," Journal of Athletic Training, vol. 50, pp. 366-377, Apr. 2015.

[41] K. E. Wilk, R. A. Williams, J. R. Dugas, E. L. Cain, and J. R. Andrews, "Current concepts in the assessment and rehabilitation of the thrower' s shoulder," Operative Techniques in Sports Medicine, vol. 24, pp. 170180, Sep. 2016.

[42] P. Larsson, P. Olofsson, E. Jakobsson, L. Burlin and K. Henriksson-Larsén, "Physiological predictors of performance in cross-country skiing from treadmill tests in male and female subjects," Scandinavian Journal of Medicine and Science in Sports, vol. 12, pp. 347-353, Dec. 2001.

[43] H. S. Sodhi, "Sports anthropometry: A kinanthropometric approach," ANOVA Publications: Mohali, 1991.

[44] A. Sharma, V. Tripathi, and S. Koley, "Correlations of anthropometric characteristics with physical fitness tests in Indian professional hockey players," Journal of Human Sport and Exercise, vol. 7, pp. 698-705, Sept. 2012.

[45] B. Podlivaev, "Model wrestlers in freestyle women's wrestling," International Journal of Wrestling Science, vol. 5, pp. 22-27, Jan. 2015.

[46] O. R. Farley, C. R. Abbiss, and J. M. Sheppard, "Testing protocols for profiling of surfers' anaerobic and aerobic fitness: A review," Strength and Conditioning Journal, vol. 38, pp. 52-65, Oct. 2016.

[47] A. Moreira, J. C. Bilsborough, C. J. Sullivan, M. Cianciosi, M. S. Aoki, and A. J. Coutts, "Training periodization of professional australian football players during an entire Australian Football League season," International Journal of Sports Physiology and Performance, vol. 10, pp. 566-571, Jul. 2015.

[48] S. A. Taha, A. R. I. Akl, and M. A. Zayed, "Electromyographic analysis of selected upper extremity muscles during jump throwing in handball," American Journal of Sports Science, vol. 3, pp. 79-84, 2015.

[49] T. J. Suchomel, S. Nimphius, and M. H. Stone, "The importance of muscular strength in athletic performance," Sports Medicine, vol. 46, pp. 1419-1449, Feb. 2016.

[50] M. R. Abdullah, R. M. Musa, A. B. Maliki, N. A. Kosni, and P. K. Suppiah, "Role of psychological factors on the performance of elite soccer players," Journal of Physical Education and Sport, vol. 1, pp. 170-176, Mar. 2016 